\title{
THE RESIDUAL FINITENESS OF CERTAIN ONE-RELATOR GROUPS
}

\author{
R. B. J. T. ALLENBY, L. E. MOSER AND C. Y. TANG ${ }^{1}$
}

ABSTRACT. We prove that the groups $\left\langle a, b ;\left(a^{-1} b^{l} a b^{m}\right)^{t}\right\rangle$, where $l, m, t \in Z$ and $t>2$ are residually finite $\left({ }_{R} F\right)$, thus establishing a conjecture of $\mathbf{G}$. Baumslag [Bull. Amer. Math. Soc. 73 (1967), 618-620].

\section{Preliminaries.}

LemMa 1. Let $G=\left\langle x, y ;\left(x^{t} y^{m}\right)^{t}\right\rangle$. Then if $g \in G \backslash\langle x\rangle$ there exists a homomorphism $\tau$ of $G$ onto a finite group $R$ such that $g \tau \notin\langle x \tau\rangle$ in $R$.

Proof. Let $T=\left\langle y, z ; z^{t}\right\rangle$. Then $T$ is LERF [5, p. 359]. Putting $u=z y^{-m}$ we get $T=\left\langle y, u y^{m} ;\left(u y^{m}\right)^{t}\right\rangle=\left\langle y, u ;\left(u y^{m}\right)^{t}\right\rangle$. Now form the generalized free product (g.f.p.)

$$
\langle x\rangle \underset{x^{l}=u}{*}\left\langle u, y ;\left(u y^{m}\right)^{t}\right\rangle=\left\langle x, y ;\left(x y^{m}\right)^{t}\right\rangle=G .
$$

Suppose $g \in G \backslash\langle x\rangle$. Then $g$ can be expressed as a product $\left(y_{1}\right) x^{i_{1}} \cdots x^{i_{r}}\left(y_{r+1}\right)$ where each $x^{i_{k}} \notin\left\langle x^{l}\right\rangle$ and each $y_{k} \in T \backslash\left\langle x^{l}\right\rangle$. Since $T$ is LERF we can find for each $y_{k}$ a normal subgroup $N_{k}$ of finite index in $T$ such that $y_{k} N_{k} \notin\left\langle x^{l} N_{k}\right\rangle$. The intersection $N$ of all these $N_{k}$ is another normal subgroup of finite index in $T$ such that $y_{k} N \notin\left\langle x^{l} N\right\rangle$ for all (the finitely many) $k$.

Form the generalized free product

$$
S=\langle x\rangle /(\langle x\rangle \cap N) \underset{\left\langle x^{\prime}\right\rangle /\left(\left\langle x^{\prime}\right\rangle \cap N\right)}{*} T / N .
$$

Letting bars denote images under the natural map from $G$ onto $S$ we see by a "form of word" argument that $\bar{g} \notin\langle\bar{x}\rangle$ in $S$. But $S$ is residually finite [3, p. 194 ] and so one easily finds a finite homomorphic image $R$ of $S$ in which the image $\bar{g}$ lies outside the image of $\langle\bar{x}\rangle$.

Clearly this result extends easily to

Corollary 2. Let $G=\left\langle x, y ;\left(x^{l} y^{m}\right)^{t}\right\rangle$. Given $g_{1}, \ldots, g_{r} \in G \backslash\langle x\rangle$ and $h_{1}, \ldots, h_{s} \in G \backslash\langle y\rangle$ there exists a normal subgroup $N^{*}$ of finite index in $G$ such that $g_{i}\langle x\rangle \cap N^{*}=h_{j}\langle y\rangle \cap N^{*}=\varnothing$.

Received by the editors November 3, 1978.

AMS (MOS) subject classifications (1970). Primary 20E25, 20F05; Secondary $20 \mathrm{E} 30$.

Key words and phrases. Generalized free product, HNN extension, residually finite, LERF.

${ }^{1}$ The work of the third author was partly supported by Canadian National Research Council Grant No. A-4064. 
As a corollary of this we have

Corollary 3. $G \in{ }_{R} F$.

Proof. Let $1 \neq g \in G$. If $g \in\langle y\rangle$ then $g \notin\langle x\rangle$ and so there exists $N^{*}$ as in Corollary 2 such that $g\langle x\rangle \cap N^{*}=\varnothing$. In particular $g$ is not in $N^{*}$. A similar proof holds if $g \in G \backslash\langle y\rangle$ (whether $g$ is in $\langle x\rangle$ or not).

LEMMA 4. Let $G=\left\langle x, y ;\left(x^{l} y^{m}\right)^{t}\right\rangle$. Then given $r \in Z$, where $r$ is any multiple of $l$ and of $m$, there exists $N \triangleleft G$ such that $|G: N|\langle\infty, N \cap\langle x\rangle=$ $\left\langle x^{r}\right\rangle$ and $N \cap\langle y\rangle=\left\langle y^{r}\right\rangle$.

PROof. Consider the group $H=\left\langle g, h ;(g h)^{t}, g^{\alpha}, h^{\beta}\right\rangle$ where $r=\alpha l=\beta m$. Then $H \in{ }_{R} F[4$, p. 425]. Thus $H$ has, as a homomorphic image, the finite group $K=\left\langle g, h ;(g h)^{t}, g^{\alpha}, h^{\beta}, w_{i}(g, h)\right\rangle$ in which $g, h$ have orders $\alpha, \beta$ exactly.

Now form the g.f.p.

$$
\left\langle x ; x^{l \alpha}\right\rangle \underset{x^{l}=g}{*} K=\left\langle x, h ;\left(x^{l} h\right)^{\ell}, x^{\alpha l}, h^{\beta}, w_{i}\left(x^{l}, h\right)\right\rangle=L,
$$

say. Now $L \in{ }_{R} F$ (being a g.f.p. of finite groups) and so has a finite homomorphic image

$$
M=\left\langle x, h ;\left(x^{l} h\right)^{t}, x^{\alpha l}, h^{\beta}, w_{i}\left(x^{l}, h\right), w_{i}^{\prime}(x, h)\right\rangle,
$$

say, in which $x$ has order $\alpha l$ and $h$ has order $\beta$. Now form

$$
U=M \underset{h=y^{m}}{*}\left\langle y ; y^{m \beta}\right\rangle=\left\langle x, y ;\left(x y^{m}\right)^{t}, x^{r}, y^{r}, w_{i}\left(x^{l}, y^{m}\right), w_{i}^{\prime}\left(x, y^{m}\right)\right\rangle .
$$

This too has a finite homomorphic image

$$
V=\left\langle x, y ;\left(x y^{m}\right)^{t}, x^{r}, y^{r}, w_{i}\left(x^{l}, y^{m}\right), w_{i}^{\prime}\left(x, y^{m}\right), w_{i}^{\prime \prime}(x, y)\right\rangle
$$

in which $x$ and $y$ both have order $r$, exactly. Clearly $V$ is a homomorphic image (under $\psi$, say) of $G$ and if we set $N=\operatorname{ker} \psi: G \rightarrow V$ we see that $N \cap\langle x\rangle=\left\langle x^{r}\right\rangle, N \cap\langle y\rangle=\left\langle y^{r}\right\rangle$ as required.

\section{The main theorem. ${ }^{2}$}

TheOREM 5. Let $l, m, t \in Z$ with $t \geqslant 2$. Then $\left\langle a, b ;\left(a^{-1} b^{l} a b^{m}\right)^{t}\right\rangle \in{ }_{R} F$.

Proof. The given group is well known to be an HNN extension with base group the ${ }_{R} F$ group $B=\left\langle b_{0}, b_{1} ;\left(b_{1}^{l} b_{0}^{m}\right)^{t}\right\rangle$. Further the action of " $a$ " on this group is to conjugate $b_{1}$ onto $b_{0}$. Thus there is an isomorphism $\phi$ from $\left\langle b_{1}\right\rangle$ onto $\left\langle b_{0}\right\rangle$ coinciding with this action. Thus all the conditions set out in 4.1 of [1] are satisfied, their " $A$ ", “ $H$ ", " $K$ " being our $B,\left\langle b_{1}\right\rangle,\left\langle b_{0}\right\rangle$, respectively. Corollary 2 shows that condition 4.1(a) of [1] holds and if $N^{*}$ does not satisfy

\footnotetext{
${ }^{2}$ This theorem was also proved by B. Baumslag and F. Levin several months earlier. The proof given here was done completely independently using an entirely different approach. It is much shorter and perhaps a little crisper. In a communication with $\mathbf{G}$. Baumslag he mentioned that he has also obtained a similar result with a more involved proof.
} 
condition 4.1(b) immediately one readily amends $N^{*}$ by intersecting it with a suitable $N$ as given by Lemma 4 .

\section{REFERENCES}

1. B. Baumslag and M. Tretkoff, Residually finite HNN extensions, Comm. Algebra 6 (1978), 179-194.

2. G. Baumslag, Residually finite one-relator groups, Bull. Amer. Math. Soc. 73 (1967), 618-620.

3. On the residual finiteness of generalised free products of nilpotent groups, Trans. Amer. Math. Soc. 106 (1963), 193-209.

4. $\ldots$ On generalised free products, Math. Z. 78 (1962), 423-438.

5. R. G. Burns, On finitely generated subgroups of free products, J. Austral. Math. Soc. 12 (1971), 358-364.

DePARTMENT OF MATHEMATICS, UNIVERStTy OF LeEDS, LeEDS, LS2 9JT, ENGLAND

Department of Mathematics, California State University, Hayward, California 94542

Department of PUre Mathematics, University of Waterloo, Waterloo, Ontario, Canada 